\title{
ATIVIDADE RESIDUAL IN VITRO DO PELO DE COBERTURA DE CÃES TRATADOS COM DINOTEFURAN SOBRE LARVAS E ADULTOS DE Ctenocephalides felis felis (BOUCHÉ, 1835) (SIPHONAPTERA: PULICIDAE)*
}

\author{
THAÍS R. CORREIA ${ }^{1}$; VANESSA P. DA CRUZ ${ }^{2}$; FRANCISCO DE A. RIBEIRO ${ }^{2}$; RAQUEL M. P. S. MELO ${ }^{1}$; \\ JULIO I. FERNANDES ${ }^{3}$; GUILHERME G. VEROCAI ${ }^{3}$; FABIO B. SCOTT ${ }^{4}$
}

\begin{abstract}
CORREIA, T.R.; CRUZ, V.P. DA; RIBEIRO, F. DE A.; MELO, R.M.P.S.; FERNANDES, J.I.; VEROCAI, G.G.; SCOTT, F.B. [Residual activity in vitro of treated dog's hair coat with dinotefuran on larvae and adults of Ctenocephalides felis felis (Bouché, 1835) (Siphonaptera: Pulicidae)]. Atividade residual in vitro do pêlo decobertura de cães tratados com dinotefuran sobre larvas e adultos de Ctenocephalides felis felis (Bouché, 1835) (Siphonaptera: Pulicidae). Revista Brasileira de Parasitologia Veterinária, v. 17, n.4, p.195-199, 2008 Curso de Pós-graduação em Ciências Veterinárias, Universidade Federal Rural do Rio de Janeiro, BR 465, Km 7 , Seropédica, RJ 2389-000, Brasil. E-mail: thaisrca@gmail.com

The aim of this study was to evaluate the residual activity of treated dog's hair coat with dinotefuran on larvae and adults of Ctenocephalides felis felis. Three female Beagle dogs were used. One female dog was treated with 0.834\% dinotefuran spray, the second was treated with 30\% dinotefuran strip-on and the third was not treated. Some areas of dog's hair were clipped on days 2, 9, 16, 23, 30, 37 and 44 after treatment. For the evaluation of adulticidal and larvicidal activities, flea adults and larvae from the laboratory colony were used. Six repetitions were used with 10 samples of each flea stage per day, placed in assay tubes. In each repetition we added $0.02 \mathrm{~g}$ of treated or untreated dog's hair and larval diet for the immature stage. Flea adult assay was evaluated with 24 hours and the flea larvae 20 days after treatment. The residue on hair coat of both treated dogs showed efficacy on the flea larvae control for 44 days. Regarding the flea adults control the spray and strip-on formulations showed efficacy until days 16 and 23, respectively.
\end{abstract}

KEY WORDS: Cat flea, stages, neonicotinoid.

\section{RESUMO}

O objetivo deste trabalho foi avaliar a atividade in vitro do resíduo em pelo de cobertura de cães tratados com dinotefuran sobre larvas e adultos de Ctenocephalides felis felis. Foram utilizadas três cadelas da Raça Beagle: a n ${ }^{0} 1$ foi tratada com uma formulação spray de dinotefuran a $0,834 \%$, a $\mathrm{n}^{\circ} 2$ com uma formulação "strip-on"de dinotefuran a 30\% e a n ${ }^{\circ} 3$ foi mantida sem tratamento. Pequenas áreas foram tricotomizadas nos dias 2, 9, 16, 23, 30, 37 e 44 após o tratamento. Para avaliação

\footnotetext{
*Sob auspícios do CNPq e FAPERJ

${ }^{1}$ Curso de Pós-graduação em Ciências Veterinárias (CPGCV), Universidade Federal Rural do Rio de Janeiro (UFRRJ), BR 465, Km 7, Seropédica, RJ 2389-000, Brasil. Bolsista FAPERJ. E-mail: thaisrca@gmail.com

${ }^{2}$ Curso de Medicina Veterinária, UFRRJ, Seropédica, RJ. E-mail: fran.ribeirovet@gmail.com, nessinhavieira@yahoo.com.br

${ }^{3}$ CPGCV, UFRRJ. E-mail: fernandesji@ufrrj.br, verocai@ufrrj.br, Bolsistas CNPq

${ }^{4}$ Departamento de Parasitologia Animal, Instituto de Veterinária, UFRRJ, E-mail: scott@ufrrj.br.
}

adulticida e larvicida, foram utilizados pulgas de uma colônia mantida em laboratório. Seis repetições com cada uma contendo 10 exemplares de C. f. felis, acondicionados em tubos de ensaio, da etapa correspondente foram utilizados por dia de desafio. Em cada repetição foi adicionado 0,02 gramas de pelo de cada área tricotomizada e dieta na etapa de larvas. O material da etapa adulto foi avaliado num período de 24 horas, o da etapa larvas foi avaliado após 20 dias de cada desafio. O resíduo no pêlo de cães tratados com ambas as formulações de dinotefuran foi eficaz no controle de larvas por um período de 44 dias, e no controle de adultos as formulações spray e a "strip-on" foram eficazes por um período de 16 e 23 dias, respectivamente.

PALAVRAS-CHAVE: Pulga do gato, formas evolutivas, neonicotinóide.

\section{INTRODUÇÃO}

Descoberto em 1998, o dinotefuran [(RS)-1-metil-2-nitro3-(tetrahidro-3-furil-metil) guanidina] é um dos mais recentes 
neonicotinóides, possuindo uma alta atividade inseticida contra insetos das ordens Hemiptera, Coleoptera, Diptera, Dictyoptera e Thysanoptera (WAKITA et al., 2003). São denominados neonicotinóides, pois se ligam agonisticamente aos receptores nicotínicos pós-sinápticos de acetilcolina, afetando as sinapses no sistema nervoso central dos insetos (KAGABU, 1997). A segurança e a eficácia dos neonicotinóides têm sido atribuídas, em parte, pela alta seletividade destes compostos pelos receptores nicotínicos de acetilcolina dos insetos quando comparados aos dos mamíferos (MATSUDA et al., 2001; TOMIZAWA; CASIDA, 2003). Estudos toxicológicos e ecotoxicológicos demonstraram que o dinotefuran tem uma toxicidade muito baixa para mamíferos, aves e animais aquáticos, e para o ambiente (WAKITA et al., 2005).

A pulga Ctenocephalides felis felis é um dos ectoparasitos mais observados em cães e gatos. Está relacionada com a transmissão de diversos patógenos para os animais, inclusive o homem. (DRYDEN; RUST, 1994). Diversos grupamentos químicos são empregados em seu controle, dentre eles, o imidacloprid, o nitempiram e o acetamiprid, todos neonicotinóides. Não existem relatos em literatura sobre o uso do dinotefuran no controle de ectoparasitos dos animais domésticos.

O presente trabalho teve como objetivo avaliar a atividade in vitro do resíduo em pelo de cobertura de cães tratados com o neonicotinóide dinotefuran sobre larvas e adultos de C.f. felis.

\section{MATERIAL E MÉTODOS}

Para avaliar a atividade residual do dinotefuran no pelo de cobertura de cães tratados foi seguida a metodologia proposta por Mehlhorn et al. (2001), porém com algumas modificações. Foram utilizadas três cadelas adultas da raça Beagle do Canil de Experimentação do Laboratório de Quimioterapia Experimental em Parasitologia Veterinária (LQEPV), do Departamento de Parasitologia Animal, Instituto de Veterinária da Universidade Federal Rural do Rio de Janeiro. As formulações experimentais testadas foram cedidas pelo Laboratório Sespo Indústria e Comércio Ltda - Divisão Vetbrands ${ }^{\mathrm{TM}}$ Saúde Animal (Ceva Santé Animale). O animal no 1 foi tratado com uma formulação spray contendo dinotefuran a $0,834 \%$ na dosagem de três jatos $(1 \mathrm{ml} /$ jato) por quilo de peso corpo$\mathrm{ral}$, correspondendo a $25 \mathrm{mg} / \mathrm{kg}$. $\mathrm{O}$ animal $\mathrm{n}^{\circ} 2$ foi tratado com uma formulação "strip-on" contendo dinotefuran a 30\% na dosagem de $1 \mathrm{ml}$ para cada $10 \mathrm{~kg}$ de peso corporal, correspondendo a $30 \mathrm{mg} / \mathrm{kg}$ de peso corporal. O animal no 3 foi mantido como controle, sem tratamento. Quarenta e oito horas após o tratamento, os animais foram submetidos à tricotomia em regiões distintas do corpo (cernelha, dorso, base da cauda, ventre e lados direito e esquerdo). Os pelos tricotomizados de cada região foram acondicionados em placas de Petri descartáveis, homogenizados e devidamente identificados com o dia de desafio, o nome do animal e o grupo ao qual pertencia. Os desafios após o tratamento foram realizados nos dias $+2,+9,+16,+23,+30,+37$ e +44 , para larvas; para adultos nos dias $+2,+9,+16,+23,+30$, seguindose o mesmo procedimento acima descrito. Foram utilizadas seis repetições para cada grupo de tratamento com 10 larvas e 10 adultos de C. f. felis oriundos da colônia mantida nas dependências do LQEPV, por dia de desafio. Em cada tubo de ensaio foram adicionados 0,02 gramas de pelos de cobertura homogenizados correspondente a cada tratamento. Nos tubos contendo larvas também foi adicionado meio grama de dieta para manutenção larval (CORREIA et al., 2003). Para a avaliação da atividade adulticida, o critério utilizado foi a motilidade, ou seja, pulgas que tivessem qualquer tipo de movimentação foram consideradas vivas. O material foi avaliado nos períodos de tempo de 10 minutos, 30 minutos, duas, oito, 16 e 24 horas. Para a avaliação da atividade larvicida, o material foi fixado em álcool $70^{\circ} \mathrm{GL}, 20$ dias após o dia de desafio, e avaliado com o auxílio de microscópio estereoscópico. A eficácia foi calculada para cada forma evolutiva e para cada dia de desafio, através da seguinte fórmula:

Tabela 1. Eficácia inseticida residual in vitro do pelo de cobertura de cães tratados com duas formulações distintas de dinotefuran, uma spray a 0,834\% (25mg/kg) e outra "stripon" a $30 \%$ (30 mg/kg), no controle de adultos de Ctenocephalides felis felis, dois dias após o tratamento.

\begin{tabular}{|c|c|c|c|c|c|c|}
\hline \multirow[t]{2}{*}{$\begin{array}{l}\text { Grupos/ } \\
\text { Repetições }\end{array}$} & \multicolumn{6}{|c|}{$\begin{array}{l}\text { Número de adultos vivos nos diferentes } \\
\text { períodos de avaliação }\end{array}$} \\
\hline & $10 \mathrm{~min}$ & $30 \mathrm{~min}$ & $2 \mathrm{~h}$ & $8 \mathrm{~h}$ & $16 \mathrm{~h}$ & $24 \mathrm{~h}$ \\
\hline \multicolumn{7}{|l|}{ Controle } \\
\hline 1 & 10 & 10 & 10 & 10 & 10 & 10 \\
\hline 2 & 10 & 10 & 10 & 10 & 10 & 10 \\
\hline 3 & 10 & 10 & 10 & 9 & 9 & 9 \\
\hline 4 & 10 & 10 & 10 & 9 & 8 & 8 \\
\hline 5 & 10 & 10 & 10 & 10 & 10 & 10 \\
\hline 6 & 10 & 10 & 10 & 10 & 10 & 10 \\
\hline Média $\pm d p^{*}$ & $10^{\mathrm{a}} \pm 0$ & $10^{a} \pm 0$ & $10^{a} \pm 0$ & $9,7^{a} \pm 0,5$ & $9,5^{a} \pm 0,8$ & $9,5^{a} \pm 0,8$ \\
\hline \multicolumn{7}{|l|}{ Spray } \\
\hline 1 & 10 & 10 & 6 & 1 & 0 & 0 \\
\hline 2 & 10 & 10 & 5 & 1 & 1 & 0 \\
\hline 3 & 10 & 10 & 5 & 2 & 1 & 0 \\
\hline 4 & 10 & 10 & 6 & 2 & 1 & 0 \\
\hline 5 & 10 & 10 & 6 & 1 & 0 & 0 \\
\hline 6 & 10 & 10 & 5 & 3 & 0 & 0 \\
\hline Média \pm dp & $10^{\mathrm{a}} \pm 0$ & $10^{a} \pm 0$ & $5,5^{b} \pm 0,5$ & $1,7^{\mathrm{b}} \pm 0,8$ & $0,5^{b} \pm 0,5$ & $0^{\mathrm{b}} \pm 0$ \\
\hline Eficácia & $0 \%$ & $0 \%$ & $45 \%$ & $82,5 \%$ & $94,7 \%$ & $100 \%$ \\
\hline \multicolumn{7}{|l|}{ "Strip-on" } \\
\hline 1 & 10 & 10 & 8 & 1 & 0 & 0 \\
\hline 2 & 10 & 10 & 7 & 3 & 1 & 0 \\
\hline 3 & 10 & 10 & 6 & 4 & 0 & 0 \\
\hline 4 & 10 & 10 & 8 & 1 & 0 & 0 \\
\hline 5 & 10 & 10 & 8 & 0 & 0 & 0 \\
\hline 6 & 10 & 10 & 9 & 3 & 1 & 0 \\
\hline Média \pm dp & $10^{a} \pm 0$ & $10^{\mathrm{a}} \pm 0$ & $7,7^{\mathrm{a}} \pm 1$ & $2,0^{\mathrm{b}} \pm 1,6$ & $0,3^{b} \pm 0,5$ & $0^{\mathrm{b}} \pm 0$ \\
\hline Eficácia (\%) & $0 \%$ & $0 \%$ & $23 \%$ & $79,4 \%$ & $96,8 \%$ & $100 \%$ \\
\hline
\end{tabular}

Médias com letras iguais nas colunas não diferem significativamente $(P>0,05) ;{ }^{*} d p=$ desvio padrão 
Tabela 2. Eficácia inseticida residual in vitro do pelo de cobertura de cães tratados com duas formulações distintas de dinotefuran, uma spray a 0,834\% $(25 \mathrm{mg} / \mathrm{kg})$ e outra "stripon" a $30 \%$ (30 mg/kg), no controle de adultos de Ctenocephalides felis felis, nove dias após o tratamento.

\begin{tabular}{|c|c|c|c|c|c|c|}
\hline \multirow[t]{2}{*}{$\begin{array}{l}\text { Grupos/ } \\
\text { Repetições }\end{array}$} & \multicolumn{6}{|c|}{$\begin{array}{l}\text { Número de adultos vivos nos diferentes } \\
\text { períodos de avaliação }\end{array}$} \\
\hline & $10 \mathrm{~min}$ & $30 \mathrm{~min}$ & $2 \mathrm{~h}$ & $8 \mathrm{~h}$ & $16 \mathrm{~h}$ & $24 \mathrm{~h}$ \\
\hline \multicolumn{7}{|l|}{ Controle } \\
\hline 1 & 10 & 10 & 10 & 10 & 10 & 10 \\
\hline 2 & 10 & 10 & 10 & 10 & 9 & 9 \\
\hline 3 & 10 & 10 & 10 & 10 & 10 & 10 \\
\hline 4 & 10 & 10 & 10 & 10 & 10 & 10 \\
\hline 5 & 10 & 10 & 10 & 10 & 9 & 9 \\
\hline 6 & 10 & 10 & 10 & 10 & 10 & 10 \\
\hline Média $\pm d p^{*}$ & $10^{a} \pm 0$ & $10^{\mathrm{a}} \pm 0$ & $10^{a} \pm 0$ & $10^{a} \pm 0$ & $9,7^{a} \pm 0,5$ & $9,7^{a} \pm 0,5$ \\
\hline \multicolumn{7}{|l|}{ Spray } \\
\hline 1 & 10 & 7 & 5 & 3 & 1 & 0 \\
\hline 2 & 10 & 9 & 6 & 2 & 1 & 0 \\
\hline 3 & 9 & 9 & 5 & 3 & 2 & 1 \\
\hline 4 & 10 & 9 & 3 & 2 & 0 & 0 \\
\hline 5 & 10 & 10 & 6 & 3 & 3 & 0 \\
\hline 6 & 10 & 10 & 6 & 3 & 1 & 0 \\
\hline Média \pm dp & $9,8^{a} \pm 0,4$ & $49,0^{a} \pm 1,1$ & $5,2^{b} \pm 1,2$ & $2,7^{b} \pm 0,5$ & $1,3^{\mathrm{b}} \pm 1$ & $0,2^{b} \pm 0,4$ \\
\hline Eficácia & $2,0 \%$ & $10 \%$ & $48 \%$ & $73 \%$ & $87 \%$ & $97,9 \%$ \\
\hline \multicolumn{7}{|l|}{ "Strip-on" } \\
\hline 1 & 10 & 10 & 5 & 3 & 3 & 0 \\
\hline 2 & 10 & 10 & 5 & 2 & 0 & 0 \\
\hline 3 & 10 & 9 & 7 & 1 & 0 & 0 \\
\hline 4 & 10 & 10 & 7 & 2 & 1 & 0 \\
\hline 5 & 10 & 9 & 7 & 2 & 0 & 0 \\
\hline 6 & 10 & 10 & 7 & 4 & 3 & 0 \\
\hline Média $\pm d p$ & $10^{a} \pm 0$ & $9,7^{a} \pm 0,5$ & $6,3^{b} \pm 1$ & $2,3^{b} \pm 1$ & $1,2^{b} \pm 1,5$ & $0^{\mathrm{b}} \pm 0$ \\
\hline Eficácia & $0 \%$ & $3 \%$ & $37 \%$ & $77 \%$ & $88 \%$ & $100 \%$ \\
\hline
\end{tabular}

Médias com letras iguais nas colunas não diferem significativamente $(P>0,05) ;{ }^{*} \mathrm{dp}=$ desvio padrão

Eficácia $=[$ (número médio da forma evolutiva viva do grupo controle - número médio da forma evolutiva viva do grupo tratado)/ (número médio da forma evolutiva viva do grupo controle)] x 100.

Todos os dados foram submetidos a transformação logarítmica [ $\log n(n+1)]$, posteriormente à análise de variância (ANOVA) e ao teste de significância de Tukey, quando a diferença entre as médias testadas foram estatisticamente significantes em nível de 95\%. No caso de comparação entre dois grupos apenas, os dados foram submetidos ao teste t para amostras independentes, após transformação logarítmica [log $n(n+1)]$ (SAMPAIO, 2002; AYRES et al., 2005).

\section{RESULTADOS E DISCUSSÃO}

Os resultados da atividade adulticida podem ser observados nas Tabelas 1 a 5. As duas formulações de dinotefuran tiveram maior eficácia entre 16 e 24 horas após a exposição dos adultos aos pêlos tratados. Na prática isto pode significar que após o tratamento de cães com o dinotefuran o produto alcançará sua eficácia máxima em um período de 24 horas.
Tabela 3. Eficácia inseticida residual in vitro do pelo de cobertura de cães tratados com duas formulações distintas de dinotefuran, uma spray a $0,834 \%(25 \mathrm{mg} / \mathrm{kg})$ e outra "stripon" a $30 \%(30 \mathrm{mg} / \mathrm{kg})$, no controle de adultos de Ctenocephalides felis felis 16 dias após o tratamento.

\begin{tabular}{|c|c|c|c|c|c|c|}
\hline \multirow[t]{2}{*}{$\begin{array}{l}\text { Grupos/ } \\
\text { Repetições }\end{array}$} & \multicolumn{6}{|c|}{$\begin{array}{l}\text { Número de adultos vivos nos diferentes } \\
\text { períodos de avaliação }\end{array}$} \\
\hline & 10 min & $30 \mathrm{~min}$ & $2 \mathrm{~h}$ & $8 \mathrm{~h}$ & $16 \mathrm{~h}$ & $24 \mathrm{~h}$ \\
\hline \multicolumn{7}{|l|}{ Controle } \\
\hline 1 & 10 & 10 & 10 & 10 & 10 & 10 \\
\hline 2 & 10 & 10 & 10 & 10 & 10 & 10 \\
\hline 3 & 10 & 10 & 10 & 10 & 10 & 10 \\
\hline 4 & 10 & 10 & 10 & 10 & 10 & 10 \\
\hline 5 & 10 & 10 & 10 & 10 & 10 & 10 \\
\hline 6 & 10 & 10 & 10 & 10 & 10 & 10 \\
\hline Média $\pm d p^{\star}$ & $10^{a} \pm 0$ & $10^{a} \pm 0$ & $10^{\mathrm{a}} \pm 0$ & $10^{a} \pm 0$ & $10^{a} \pm 0$ & $10^{a} \pm 0$ \\
\hline \multicolumn{7}{|l|}{ Spray } \\
\hline 1 & 10 & 9 & 3 & 1 & 0 & 0 \\
\hline 2 & 10 & 10 & 9 & 4 & 1 & 0 \\
\hline 3 & 10 & 9 & 5 & 2 & 0 & 0 \\
\hline 4 & 10 & 8 & 5 & 2 & 0 & 0 \\
\hline 5 & 10 & 5 & 0 & 0 & 0 & 0 \\
\hline 6 & 10 & 9 & 2 & 1 & 0 & 0 \\
\hline Média $\pm d p$ & $10^{a} \pm 0$ & $8,3^{a} \pm 1,7$ & $4,0^{b} \pm 3,1$ & $1,7^{b} \pm 1,4$ & $0,2^{b} \pm 0,4$ & $0^{b} \pm 0$ \\
\hline Eficácia & $0 \%$ & $17 \%$ & $60 \%$ & $83 \%$ & $98 \%$ & $100 \%$ \\
\hline \multicolumn{7}{|l|}{ "Strip-on" } \\
\hline 1 & 10 & 6 & 0 & 0 & 0 & 0 \\
\hline 2 & 10 & 7 & 0 & 0 & 0 & 0 \\
\hline 3 & 10 & 3 & 0 & 0 & 0 & 0 \\
\hline 4 & 10 & 6 & 4 & 2 & 0 & 0 \\
\hline 5 & 10 & 1 & 0 & 0 & 0 & 0 \\
\hline 6 & 10 & 3 & 3 & 2 & 0 & 0 \\
\hline Média $\pm d p$ & $10^{\mathrm{a}} \pm 0$ & $4,3^{b} \pm 2,3$ & $1,2^{b} \pm 1,8$ & $0,7^{\mathrm{b}} \pm 1$ & $0^{b} \pm 0$ & $0^{b} \pm 0$ \\
\hline Eficácia & $0 \%$ & $57 \%$ & $88 \%$ & $93 \%$ & $100 \%$ & $100 \%$ \\
\hline
\end{tabular}

Médias com letras iguais nas colunas não diferem significativamente $(P>0,05) ; * d p=$ desvio padrão

Os resultados deste estudo demonstraram que uma formulação "strip-on" permanece por mais tempo no animal, em função da sua dispersibilidade, prolongando assim a sua atividade residual inseticida

O dinotefuran teve atividade adulticida semelhante, em ambas as formulações spray e "strip-on”, quando comparado com o imidacloprid, outro neonicotinoide, em um ensaio semelhante, no qual foi avaliada a sua atividade adulticida utilizando pêlos de cães tratados com uma formulação "spot-on" (MEHLHORN et al., 2001), no qual o autor ressalta a importância do resíduo nos pêlos que se desprendem dos animais como forma de controle ambiental das formas adultas, larvares e ovos de pulgas de cães.

Os resultados da atividade larvicida podem ser observados na Tabela 6. As formulações spray e "strip-on" de dinotefuran produziram efeitos larvicidas similares e ambas as formulações tiveram suas médias de adultos inferiores ao do grupo controle por todos os 44 dias após o tratamento. Resultados semelhantes foram obtidos por Mehlhorn et al. (2001) em um único desafio empregando o imidacloprid. 
Tabela 4. Eficácia inseticida residual in vitro do pelo de cobertura de cães tratados com duas formulações distintas de dinotefuran, uma spray a 0,834\% (25mg/kg) e outra "stripon" a $30 \%$ (30 mg/kg), no controle de adultos de Ctenocephalides felis felis, 23 dias após o tratamento.

\begin{tabular}{|c|c|c|c|c|c|c|}
\hline \multirow[t]{2}{*}{$\begin{array}{l}\text { Grupos/ } \\
\text { Repetições }\end{array}$} & \multicolumn{6}{|c|}{$\begin{array}{l}\text { Número de adultos vivos nos diferentes } \\
\text { períodos de avaliação }\end{array}$} \\
\hline & $10 \mathrm{~min}$ & $30 \mathrm{~min}$ & $2 \mathrm{~h}$ & $8 \mathrm{~h}$ & $16 \mathrm{~h}$ & $24 \mathrm{~h}$ \\
\hline \multicolumn{7}{|l|}{ Controle } \\
\hline 1 & 10 & 10 & 10 & 10 & 10 & 10 \\
\hline 2 & 10 & 10 & 10 & 10 & 10 & 10 \\
\hline 3 & 10 & 10 & 10 & 10 & 10 & 10 \\
\hline 4 & 10 & 10 & 10 & 10 & 10 & 10 \\
\hline 5 & 10 & 10 & 10 & 10 & 10 & 10 \\
\hline 6 & 10 & 10 & 10 & 10 & 10 & 10 \\
\hline Média $\pm d p^{\star}$ & $10^{a} \pm 0$ & $10^{\mathrm{a}} \pm 0$ & $10^{\mathrm{a}} \pm 0$ & $10^{\mathrm{a}} \pm 0$ & $10^{\mathrm{a}} \pm 0$ & $10^{\mathrm{a}} \pm 0$ \\
\hline \multicolumn{7}{|l|}{ Spray } \\
\hline 1 & 10 & 10 & 10 & 10 & 7 & 7 \\
\hline 2 & 10 & 10 & 10 & 10 & 9 & 9 \\
\hline 3 & 10 & 10 & 10 & 10 & 10 & 10 \\
\hline 4 & 10 & 10 & 10 & 10 & 3 & 3 \\
\hline 5 & 10 & 10 & 10 & 10 & 10 & 10 \\
\hline 6 & 10 & 10 & 10 & 10 & 7 & 7 \\
\hline Média $\pm d p$ & $10^{a} \pm 0$ & $10^{a} \pm 0$ & $10^{a} \pm 0$ & $10^{a} \pm 0$ & $7,7^{a} \pm 2,7$ & $7,7^{\mathrm{a}} \pm 2,7$ \\
\hline Eficácia & $0 \%$ & $0 \%$ & $0 \%$ & $0 \%$ & $23 \%$ & $23 \%$ \\
\hline \multicolumn{7}{|l|}{ "Strip-on" } \\
\hline 1 & 10 & 10 & 10 & 10 & 0 & 0 \\
\hline 2 & 10 & 10 & 10 & 10 & 0 & 0 \\
\hline 3 & 10 & 10 & 10 & 10 & 0 & 0 \\
\hline 4 & 10 & 10 & 10 & 10 & 0 & 0 \\
\hline 5 & 10 & 10 & 10 & 10 & 0 & 0 \\
\hline 6 & 10 & 10 & 10 & 10 & 0 & 0 \\
\hline Média & $10^{\mathrm{a}} \pm 0$ & $10^{\mathrm{a}} \pm 0$ & $10^{\mathrm{a}} \pm 0$ & $10^{\mathrm{a}} \pm 0$ & $0^{\mathrm{b}} \pm 0$ & $0^{b} \pm 0$ \\
\hline Eficácia & $0 \%$ & $0 \%$ & $0 \%$ & $0 \%$ & $100 \%$ & $100 \%$ \\
\hline
\end{tabular}

Médias com letras iguais nas colunas não diferem significativamente $(P>0,05) ;{ }^{*} d p=$ desvio padrão

Jacobs et al. (2000) apesar de terem utilizado uma metodologia distinta, mas com o mesmo objetivo, observaram que a atividade larvicida do imidacloprid em cobertores de lã onde os gatos tratados repousavam foi de $100 \%$ na primeira semana declinando para 60 e $74 \%$, nas terceira e quarta semana após o tratamento. Tais resultados foram semelhantes aos obtidos neste estudo apenas na primeira semana, e inferiores aos obtidos neste estudo que durou sete semanas com atividade larvicida entre 87 e $100 \%$. A atividade larvicida do dinotefuran pode ser considerada benéfica, pois contribui com o controle ambiental. Na ausência de larvas, e conseqüentemente de pupas e adultos recém emergidos no ambiente, os animais estariam protegidos de possíveis reinfestações pelo menos até o próximo tratamento. Esta atividade larvicida poderia ocorrer principalmente nos locais de repouso dos animais, nos quais se encontrariam a maior concentração da população ambiental de pulgas, em função do desprendimento de pelos de cobertura impregnados com inseticida.
Tabela 5. Eficácia inseticida residual in vitro do pelo de cobertura de cães tratados com duas formulações distintas de dinotefuran, uma spray a 0,834\% (25mg/kg) e outra "stripon" a $30 \%$ (30 mg/kg), no controle de adultos de Ctenocephalides felis felis, 30 dias após o tratamento.

\begin{tabular}{|c|c|c|c|c|c|c|}
\hline \multirow[t]{2}{*}{$\begin{array}{c}\text { Grupos/ } \\
\text { Repetições }\end{array}$} & \multicolumn{6}{|c|}{$\begin{array}{l}\text { Número de adultos vivos nos diferentes } \\
\text { períodos de avaliação }\end{array}$} \\
\hline & $10 \min$ & $30 \mathrm{~min}$ & $2 \mathrm{~h}$ & $8 \mathrm{~h}$ & $16 \mathrm{~h}$ & $24 \mathrm{~h}$ \\
\hline \multicolumn{7}{|l|}{ Controle } \\
\hline 1 & 10 & 10 & 10 & 9 & 8 & 8 \\
\hline 2 & 10 & 10 & 10 & 10 & 10 & 10 \\
\hline 3 & 10 & 10 & 10 & 10 & 9 & 9 \\
\hline 4 & 10 & 10 & 10 & 10 & 9 & 9 \\
\hline 5 & 10 & 10 & 10 & 10 & 10 & 10 \\
\hline 6 & 10 & 8 & 8 & 8 & 8 & 8 \\
\hline Média $\pm d p^{*}$ & $10^{\mathrm{a}} \pm 0$ & $9,7^{a} \pm 0,8$ & $9,7^{a} \pm 0,8$ & $9,5^{a} \pm 0,8$ & $9,0^{a} \pm 0,9$ & $9,0^{a} \pm 0,9$ \\
\hline \multicolumn{7}{|l|}{ Spray } \\
\hline 1 & 10 & 10 & 10 & 7 & 4 & 2 \\
\hline 2 & 10 & 10 & 10 & 9 & 9 & 6 \\
\hline 3 & 10 & 10 & 10 & 10 & 10 & 10 \\
\hline 4 & 10 & 10 & 9 & 7 & 7 & 7 \\
\hline 5 & 10 & 10 & 10 & 10 & 10 & 7 \\
\hline 6 & 10 & 10 & 10 & 10 & 7 & 5 \\
\hline Média $\pm d p$ & $10^{\mathrm{a}} \pm 0$ & $10^{a} \pm 0$ & $9,8^{a} \pm 0,4$ & $8,8^{a} \pm 1,5$ & $7,8^{\mathrm{ab}} \pm 2,3$ & $6,2^{\mathrm{ab}} \pm 2,6$ \\
\hline Eficácia & $0 \%$ & $0 \%$ & $0 \%$ & $7,4 \%$ & $13 \%$ & $31 \%$ \\
\hline \multicolumn{7}{|l|}{ "Strip-on" } \\
\hline 1 & 10 & 10 & 10 & 9 & 7 & 3 \\
\hline 2 & 10 & 10 & 10 & 6 & 5 & 3 \\
\hline 3 & 10 & 10 & 10 & 10 & 8 & 7 \\
\hline 4 & 10 & 10 & 10 & 3 & 2 & 1 \\
\hline 5 & 10 & 10 & 10 & 9 & 6 & 5 \\
\hline 6 & 10 & 10 & 10 & 7 & 5 & 3 \\
\hline Média \pm dp & $10^{\mathrm{a}} \pm 0$ & $10^{\mathrm{a}} \pm 0$ & $10^{\mathrm{a}} \pm 0$ & $7,3^{\mathrm{a}} \pm 2,6$ & $5,5^{\mathrm{b}} \pm 2,1$ & $3,7^{\mathrm{b}} \pm 2,1$ \\
\hline Eficácia & $0 \%$ & $0 \%$ & $0 \%$ & $23,1 \%$ & $38,9 \%$ & $58,9 \%$ \\
\hline
\end{tabular}

Médias com letras iguais nas colunas não diferem significativamente $(P>0,05) ;{ }^{*} d p=$ desvio padrão

O dinotefuran apresenta uma excelente propriedade inseticida, além de proporcionar um excelente controle frente à ampla variedade de pragas que acometem muitos tipos de culturas. Essas características fazem com que o dinotefuran seja uma molécula promissora para ser empregada no controle de parasitos de importância em saúde pública, visto que muitos deles possuem resistência ou resistência múltipla a outros grupamentos de inseticidas (WAKITA et al., 2005), mostrando-se como uma alternativa terapêutica no controle destes parasitos.

\section{CONCLUSÃO}

Pelos de cobertura oriundos de cães tratados com dinotefuran nas formulações spray (0,834\%) e "strip-on”(30\%) demonstraram ter efeito inseticida sobre adultos e larvas de C. f. felis. A formulação "strip-on" teve um período residual mais duradouro para as formas adultas, enquanto que para as larvas não se observou diferença entre as duas formulações em teste. 
Tabela 6. Eficácia inseticida residual in vitro do pelo de cobertura de cães tratados com duas formulações distintas de dinotefuran, uma spray a 0,834\% $(25 \mathrm{mg} / \mathrm{kg})$ e outra "stripon" a $30 \%$ (30 mg/kg), no controle de larvas de Ctenocephalides felis felis.

\begin{tabular}{|c|c|c|c|c|c|c|c|}
\hline \multirow[t]{2}{*}{$\begin{array}{c}\text { Grupos/ } \\
\text { Repetições }\end{array}$} & \multicolumn{7}{|c|}{$\begin{array}{c}\mathrm{N}^{0} \text { de adultos emergidos } 20 \text { dias após } \\
\text { cada dia de desafio }\end{array}$} \\
\hline & +2 & +9 & +16 & +23 & +30 & +37 & +44 \\
\hline \multicolumn{8}{|l|}{ Controle } \\
\hline 1 & 9 & 10 & 9 & 10 & 9 & 10 & 9 \\
\hline 2 & 10 & 9 & 10 & 10 & 9 & 9 & 10 \\
\hline 3 & 10 & 10 & 10 & 10 & 9 & 10 & 10 \\
\hline 4 & 9 & 10 & 9 & 10 & 8 & 9 & 10 \\
\hline 5 & 9 & 9 & 9 & 10 & 9 & 10 & 10 \\
\hline 6 & 10 & 9 & 10 & 9 & 10 & 10 & 9 \\
\hline Média $\pm d p^{\star}$ & $9,5^{a} \pm 0,5$ & $9,5^{a} \pm 0,5$ & $9,5^{a} \pm 0,5$ & $9,8^{a} \pm 0,41$ & $9,0^{a} \pm 0,6$ & $9,7^{a} \pm 0,5$ & $9,7^{a} \pm 0,5$ \\
\hline \multicolumn{8}{|l|}{ Spray } \\
\hline 1 & 0 & 0 & 0 & 1 & 1 & 1 & 0 \\
\hline 2 & 0 & 0 & 0 & 1 & 1 & 0 & 0 \\
\hline 3 & 0 & 0 & 0 & 0 & 1 & 0 & 0 \\
\hline 4 & 0 & 0 & 1 & 1 & 0 & 1 & 0 \\
\hline 5 & 0 & 0 & 0 & 2 & 0 & 0 & 0 \\
\hline 6 & 0 & 0 & 0 & 2 & 1 & 0 & 0 \\
\hline Média $\pm d p$ & $0^{b} \pm 0$ & $0^{\mathrm{b}} \pm 0$ & $0,2^{\mathrm{b}} \pm 0,4$ & $1,2^{\mathrm{b}} \pm 0,8$ & $0,7^{\mathrm{b}} \pm 0,5$ & $0,3^{b} \pm 0,5$ & $0^{b}$ \\
\hline Eficácia & $100 \%$ & $100 \%$ & $97,9 \%$ & $87,7 \%$ & $92,2 \%$ & $96,9 \%$ & $100 \%$ \\
\hline \multicolumn{8}{|l|}{ "Strip-on" } \\
\hline 1 & 0 & 0 & 0 & 1 & 0 & 0 & 0 \\
\hline 2 & 0 & 0 & 1 & 0 & 0 & 0 & 0 \\
\hline 3 & 0 & 0 & 0 & 1 & 0 & 0 & 0 \\
\hline 4 & 0 & 0 & 0 & 0 & 0 & 0 & 0 \\
\hline 5 & 0 & 0 & 0 & 0 & 0 & 0 & 0 \\
\hline 6 & 0 & 0 & 0 & 0 & 0 & 0 & 0 \\
\hline Média \pm dp & $0^{\mathrm{b}} \pm 0$ & $0^{\mathrm{b}} \pm 0$ & $0,2^{\mathrm{b}} \pm 0,4$ & $0,3^{b} \pm 0,5$ & $0^{\mathrm{b}} \pm 0$ & $0^{\mathrm{b}} \pm 0$ & $0^{b} \pm 0$ \\
\hline Eficácia & $100 \%$ & $100 \%$ & $97,9 \%$ & $96,9 \%$ & $100 \%$ & $100 \%$ & $100 \%$ \\
\hline
\end{tabular}

Médias com letras iguais nas colunas não diferem significativamente $(P>0,05) ;{ }^{*} d p=$ desvio padrão

\section{REFERÊNCIAS BIBLIOGRÁFICAS}

AYRES, M.; AYRES JR, M; AYRES, D.L.; SANTOS, A.S. BioEstat 4.0 - Aplicações estatísticas nas áreas das ciências biológicas e médicas. $4^{\mathrm{a}}$ ed. Belém: Sociedade Civil Mamirauá/Imprensa Oficial do Estado do Pará, 2005. 324 p. CORREIA, T.R.; SOUZA, C.P.; FERNANDES, J.I.; MARTINS, I.V.F.; SANTOS, H.D.; SCOTT, F.B. Ciclo bio- lógico de Ctenocephalides felis felis (Bouché, 1835) (Siphonaptera, Pulicidae) a partir de diferentes dietas artificiais. Revista Brasileira de Zoociências, v. 5, n. 2, p. 153160, 2003.

DRYDEN, M.W.; RUST, M.K. The cat flea: biology, ecology and control. Veterinary Parasitology, n. 52, n. 1-2, p. 1-19, 1994.

JACOBS, D.E.; HUTCHINSON, M.J.; EWALD-HAMM, D. Inhibition of immature Ctenocephalides felis felis (Siphonaptera: Pulicidae) development in the immediate environment of cats treated with imidacloprid. Journal of Medical Entomology, v. 37, n. 2, p. 228-230, 2000.

KAGABU, S. Chloronicotinyl insecticides - discovery, application and future perspective. Reviews in Toxicology, v. 1, n. 7-8, p. 75-129, 1997.

MATSUDA, K.; BUCKINGHAM, S.D.; KLEIER, D.; RAUH, J.J.; GRAUSO, M.; SATTELLE, D.B. Neonicotinoids: insecticides acting on insect nicotinic acetylcholine receptors. Trends in Pharmacological Sciences, v. 22, n. 11, p. 573-580, 2001.

MEHLHORN, H.; HANSEN, O.; MENCKE, N. Comparative study on the effects of three insericides (fipronil, imidacloprid, selamectin) on development stages of the cat flea (Ctenocephalides felis Bouché, 1835): a light and electron microscopic analysis of in vivo and in vitro experiments. Parasitology Research, v. 87, n. 3, p. 198207, 2001.

SAMPAIO, I.B.M. Estatística aplicada à experimentação animal. $2^{\mathrm{a}}$ ed. Belo Horizonte: FEPMVZ, 2002. 265p.

TOMIZAWA, M.; CASIDA, J.E. Selective toxicity of neonicotinoids attribuable to specificity of insect and mammalian nicotinic receptors. Annual Review of Entomology, v. 48, n. 1, p. 339-364, 2003.

WAKITA, T.; KINOSHITA, K.; YAMADA, E.; YASUI, N.; KAWAHARA, N.; NAOI, A.; NAKAYA, M.; EBIHARA, K.; MATSUNO, H.; KODAKA, K. The discovery of dinotefuran: a novel neonicotinoid. Pest Management Science, v. 59, n. 9, p. 1016-1022, 2003.

WAKITA, T.; YASUI, N.; YAMADA, E.; KISHI, D. Development of a novel insecticide, dinotefuran. Journal of Pesticide Science, v. 30, n. 2, p, 122-123, 2005.

Recebido em 29 de janeiro de 2007.

Aceito para publicação em 22 de dezembro de 2008. 